Europhys. Lett., 56 (6), pp. 891-897 (2001)

\title{
Ionization and photon emission in single-bubble sonoluminescence
}

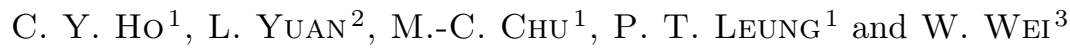 \\ 1 Department of Physics, The Chinese University of Hong Kong \\ Shatin, Hong Kong, PRC \\ 2 LSEC and Institute of Computational Mathematics \\ Academy of Mathematics and System Sciences - Academia Sinica, Beijing 100080, PRC \\ 3 Nonlinear Center, University of Science and Technology of China \\ Hefei 230026, PRC
}

(received 6 June 2001; accepted in final form 1 October 2001)

PACS. 78.60.Mq - Sonoluminescence; triboluminescence.

PACS. 47.40.-x - Compressible flows; shock and detonation phenomena.

PACS. 52.50.Lp - Plasma production and heating by shock waves and compression.

\begin{abstract}
The full set of hydrodynamic equations governing the pulsation of a sonoluminescing (SL) bubble is solved numerically taking ionization into account. We find that the hydro-thermal processes in an SL bubble are dominated by the formation of compression waves and up to $27 \%$ of first-level ionization. The ionized core is optically opaque, and the modified emitted light power and pulse widths, as well as their wavelength dependence, are in excellent agreement with experimental data.
\end{abstract}

The remarkable discovery that sound can be converted to light through an oscillating air bubble trapped in water has attracted widespread interest in single-bubble sonoluminescence (SBSL) $[1,2]$. Under certain conditions, a narrow and regular flash of light with a width of about 40-350 ps is emitted in each cycle of the bubble oscillation [3-5]. Many models have been proposed to explain the cause of light emission, and these models have to explain the spectrum of the emitted light and the wavelength independence of the pulse widths [3-5].

Previous calculations based on inviscid spherical hydrodynamics [6-8] suggested that a converging shock produced high temperature and pressure and the reflected diverging shock quenched them in picosecond time scale [7-9]. Xu et al. reported very strong multiple ionizations, up to $\mathrm{Ar}^{5+}$, based on this scenario $[10,11]$. This model could explain the emergence of a picosecond time scale as well as the large energy concentration. But the effects of transport processes, surface tension, the equation of state (EOS), and the compressibility of liquid were also largely ignored. One recent calculation that includes a temperature-dependent surface tension and thermal conduction shows that most of the trends of experimental data can be explained based on a shock-induced cold dense plasma model [12]. On the other hand, assuming a uniform bubble interior, Hilgenfeldt et al. could explain the SL spectral shape and the wavelength independence of the pulse widths using a modified blackbody radiation model that accounts for the small emissivity and absorption of a weakly ionized gas [13]. This puts the necessity of shock-induced plasma in SBSL in doubt. However, the assumed uniformity of the bubble content is unrealistic during the bubble collapse and light emission.

(C) EDP Sciences 
These models have presented rather contradictory pictures of the bubble interior during light emission. There is yet no consensus on whether an SL bubble is strongly ionized, as proposed in $[10,11]$, or only weakly so [13]. The validity of most of these models depends on the details of the hydro-thermal processes inside the bubble, and therefore a realistic hydrodynamics model is essential. Recent studies [14-18] that include parts or all of the effects of the tranport processes demonstrate that shock waves are not essential for stable SBSL. Rather, smooth compressional waves emerge naturally in an SL bubble, which raise the temperature to several $10^{4} \mathrm{~K}$ and can indeed induce chemical reactions and ionization.

We study in this paper the degrees of ionization in SBSL and its relevance to the lightemission mechanism of SBSL based on a realistic hydrodynamic calculation. Our major conclusions are: 1) shock waves are absent in the stable SBSL regime; 2) the maximum fraction of atoms ionized, calculated from either the Saha equation or a thermal bremsstrahlung model, is between $7 \%$ to $27 \%$, and $\mathrm{Ar}^{+}$is the only dominant ion; 3) because of ionization, the core of the bubble becomes optically opaque, and an opacity-corrected blackbody radiation model gives peak power, pulse widths and spectra that agree with the experimental data.

Neglecting mass diffusion, which is a much slower process $[1,2]$, the dynamics of the gas in a spherical bubble is described by the multi-species, compressible Navier-Stokes (NS) equations:

$$
\frac{\partial \boldsymbol{Q}}{\partial t}+\frac{\partial \boldsymbol{F}(\boldsymbol{Q})}{\partial x}=\boldsymbol{H}(\boldsymbol{Q})+\boldsymbol{F}_{v}(\boldsymbol{Q})+\boldsymbol{S}_{\mathrm{s}}(\boldsymbol{Q})
$$

where $x \equiv r / R(t)$ has been used to transform the equations to a fixed domain $x \in[0,1]$. Here $\boldsymbol{Q}=R(\rho \boldsymbol{f}, \rho, \rho v, \rho E)^{T}$, with $E=e+\frac{1}{2} v^{2}$ the total energy per unit mass, $\boldsymbol{f}=\left(f_{0}, \ldots, f_{L-1}, f_{e}\right)$ the mass fractions of the $(L-1)$-ionized states of the atoms and of electrons, $R, \rho, v, p, T$, and $e$ the bubble radius, density, radial velocity, pressure, temperature, and internal energy per unit mass, respectively. The inviscid fluxes are $\boldsymbol{F}=\left[\rho \boldsymbol{f}(v-x \dot{R}), \rho(v-x \dot{R}), \rho v^{2}+p-\rho v x \dot{R},(\rho E+\right.$ p) $v-\rho E x \dot{R}]^{T}$, and the spherical sources are $\boldsymbol{H}=-2 v[\rho \boldsymbol{f}, \rho, \rho v,(\rho E+p)]^{T} / x$. The diffusive transport terms are given by $\boldsymbol{F}_{v}=\left\{0,0, \partial_{x}\left(x^{2} \tau_{r r}\right)+x \tau_{r r}, \partial_{x}\left[x^{2}\left(v \tau_{r r}+\kappa \partial_{x} T / R\right]\right\}^{T} / x^{2}\right.$, where $\tau_{r r}=4 \mu\left(\partial_{x} v-v / x\right) / 3 R$ is the normal viscous stress, $\kappa$ is the coefficient of thermal conductivity, $\mu$ is the dynamic viscosity. The source term $\boldsymbol{S}_{\mathrm{s}}$ accounts for the mass production due to ionization and recombination processes.

The van der Waals EOS has been modified taking ionization into account $[10,11]$,

$$
\begin{aligned}
p & =\left(\sum_{i=0}^{L} \frac{f_{i}}{m_{i}}+\frac{f_{e}}{m_{e}}\right) \frac{k \rho T}{1-b \rho}, \\
e & =\frac{3 k T}{2}\left(\sum_{i=0}^{L} \frac{f_{i}}{m_{i}}+\frac{f_{e}}{m_{e}}\right)+k \sum_{i=1}^{L} \sum_{j=i}^{L} \frac{f_{j}}{m_{j}} T_{i},
\end{aligned}
$$

where $m_{i}$ is the mass of an atom $(i=0)$ or an ion with a charge $i, m_{e}$ is the mass of an electron, $k$ is the Boltzman constant, and $b$ is the excluded volume. We assume that an SL bubble is made up of argon gas [19]. The mass fraction of $\mathrm{Ar}^{L+}, f_{L}$, is determined by using the mass conservation of species, $f_{L}=1-f_{e}-\sum_{i=0}^{L-1} f_{i}$, and $T_{i}$ is the ionization energy of an ion with charge $i-1$. The last term in eq. (2) represents the total energy required for ionization. The maximum level of ionization $L$ is taken to be 5 here.

The net rate of change of the number density $n_{i}=f_{i} \rho / m_{i}$ of an atom or an ion with charge $i$ is given by

$$
\begin{aligned}
\dot{n}_{i}= & n_{i-1} n_{e} \alpha_{i-1 \rightarrow i}^{\text {ion }}-n_{i} n_{e} \alpha_{i \rightarrow i+1}^{\text {ion }}+n_{i+1} n_{e}\left(\alpha_{i+1 \rightarrow i}^{\text {rrec }}+\alpha_{i+1 \rightarrow i}^{\text {trec }}\right)- \\
& -n_{i} n_{e}\left(\alpha_{i \rightarrow i-1}^{\text {rrec }}+\alpha_{i \rightarrow i-1}^{\text {trec }}\right),
\end{aligned}
$$


where $\alpha_{i \rightarrow i+1}^{\text {ion }}, \alpha_{i \rightarrow i-1}^{\text {rrec }}$ and $\alpha_{i \rightarrow i-1}^{\text {trec }}$ denote the rates of ionization, radiative recombination and three-body recombination of particles with a charge of $i[20,21]$.

The NS equations are coupled to the Rayleigh-Plesset (RP) equation that describes the bubble wall motion. It has been shown that whether shocks emerge depends strongly on the form of the RP equation [17,18]. To account for the effect of the liquid compressibility better, we use a more complete RP equation that includes terms to first order in the Mach number $M \equiv \dot{R} / c_{1}$ and allows for a variable speed of sound in the liquid $c_{1}$ [22], giving

$$
\frac{1-M}{1+M} R \ddot{R}+\frac{3-M}{2(1+M)} \dot{R}^{2}=H_{\mathrm{b}}-\frac{P_{\mathrm{s}}\left(t^{\prime}\right)}{\rho_{\infty}}+\frac{t_{R} \dot{H}_{\mathrm{b}}}{1+M} .
$$

Here overdots denote time derivatives, $t_{R} \equiv R / c_{1}, t^{\prime} \equiv t+t_{R}, \rho_{\infty}$ is the ambient density, $P_{\mathrm{s}}\left(t^{\prime}\right)=-P_{\mathrm{a}} \sin \left(\omega t^{\prime}\right)$ the pressure of the sound field with frequency $\omega$ and amplitude $P_{\mathrm{a}}$, and $H_{\mathrm{b}}$ is the enthalpy of the liquid. For water, $c_{1}$ and $H_{\mathrm{b}}$ are obtained using an EOS of the modified Tait form [23]. In computing $H_{\mathrm{b}}$, the pressure $P_{\mathrm{b}}(t)$ on the liquid side of the bubble is related to the pressure $P_{\mathrm{g}}(R, t)$ on the gas side of the bubble wall via the normal stress balance condition [23]. We also solve the energy equation for the surrounding water to allow for the heat transfer across the bubble wall [17]. We solve eq. (1) using a second-order TVD scheme, the details of which can be found in ref. [17,24].

We calculate the emitted light spectrum using a blackbody radiation model refined in two ways to accommodate the effects of ionization [24]. First, the presence of ionized plasma greatly reduces the photon mean free path, and hence we divide the bubble into a strongly absorbing core and a weakly absorbing outershell, according to the wavelength-dependent mean free path $l(r, t, \lambda)$. We define the radius of the "black" core $R_{\mathrm{c}}$ to be the point where $l$ equals a fraction of the bubble radius, $l\left(R_{\mathrm{c}}, t, \lambda\right)=R(t) / n_{\mathrm{co}}$. Furthermore, we define an inner core whose radius $R_{\mathrm{ci}}$ is related to the bubble radius in a similar manner: $l\left(R_{\mathrm{ci}}, t, \lambda\right)=$ $R(t) / n_{\mathrm{ci}}$, with $n_{\mathrm{ci}}>n_{\mathrm{co}}$. The core surface temperature $T\left(r=R_{\mathrm{c}}\right)$ is increased because photons emitted in the inner core are absorbed by the surface layer of the core. We treat this effect approximately by redistributing the radiation energy of the inner core to the gas in the surface layer $R_{\mathrm{ci}}<r<R_{\mathrm{c}}$. In practice, we choose $n_{\mathrm{ci}}, n_{\mathrm{co}}$ to be 8 and 4 , respectively, which gives a good fit to data, but our results are hardly changed if they are 10 and 5 instead. Note that the core size and the mean free paths are smaller than the wavelengths of the emitted light. A careful study of the intricate effects this may cause is certainly interesting, and we have considered them in a recent paper for a static bubble [25]. However, it is beyond the scope of the present paper to incorporate these finite-size effects into the case of a rapidly oscillating bubble.

Secondly, the surface emission from the "black" core is further subjected to the absorption effect of the "grey" outershell, as described by a damped intensity in the small-opacity approximation. The total spectral radiance (power emitted per wavelength interval) of the bubble content at wavelength $\lambda$ is then given by [26]

$$
\begin{aligned}
P_{\lambda}^{\mathrm{Pl}}(t)= & \int_{R_{\mathrm{c}}}^{R} 16 \pi \kappa_{\lambda}^{\mathrm{tot}}(r, t) R_{\lambda}^{\mathrm{Pl}}(r, t) \exp \left[-\int_{r}^{R} \kappa_{\lambda}^{\mathrm{tot}}\left(r^{\prime}\right) \mathrm{d} r^{\prime}\right] \Theta(r) r^{2} \mathrm{~d} r+ \\
& +4 \pi R_{\mathrm{c}}^{2} R_{\lambda}^{\mathrm{Pl}}\left(R_{\mathrm{c}}, t\right) \exp \left[-\int_{R_{c}}^{R} \kappa_{\lambda}^{\mathrm{tot}}\left(r^{\prime}\right) \mathrm{d} r^{\prime}\right]
\end{aligned}
$$

where

$$
R_{\lambda}^{\mathrm{Pl}}(T(r, t))=\frac{2 \pi h c^{2}}{\lambda^{5}} \cdot[\exp [h c / \lambda k T]-1]^{-1}
$$



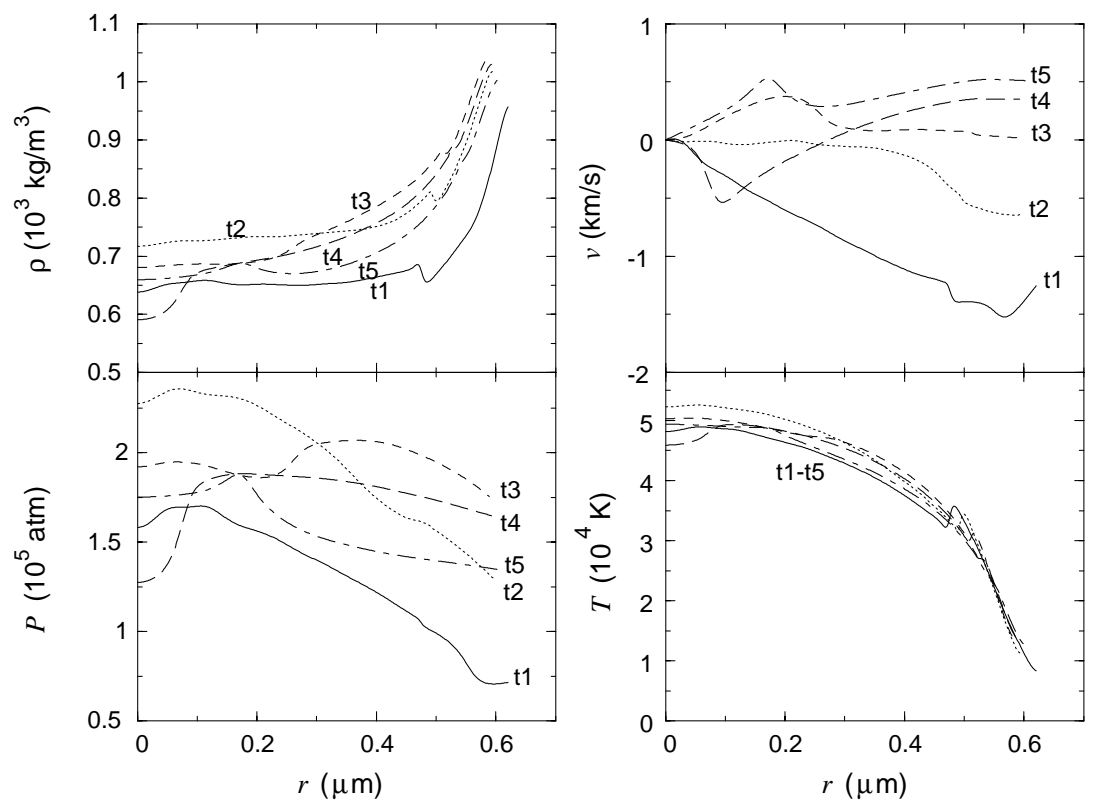

Fig. 1 - Snapshots of the spatial profiles of density, velocity, pressure, and temperature for $P_{\mathrm{a}}=$ $1.325 \mathrm{~atm}$ around the instant of maximum compression.

is the spectral emissive power, and the overall absorption coefficient $\kappa_{\lambda}^{\text {tot }}$ is greater for red light than for UV [24], and $\Theta(r)=\left[1+\sqrt{1-\left(R_{\mathrm{c}} / r\right)^{2}}\right] / 2$ is a correction factor to account for the blocking of photons by the black core.

We assume $P_{0}=1 \mathrm{~atm}, T_{0}=300 \mathrm{~K}, \omega / 2 \pi=26.4 \mathrm{kHz}$, and standard values for other parameters [17]. We choose $P_{\mathrm{a}}, R_{0}$ in the stable range of SL [27], specifically, $P_{\mathrm{a}}=$ $1.275,1.3,1.325,1.35$ atm and $R_{0}=2.6,4,4.7,5.4 \mu \mathrm{m}$, respectively. We also calculate for $P_{\mathrm{a}}=1.23 \mathrm{~atm}$ and $R_{0}=2.2 \mu \mathrm{m}$ to study the onset of SBSL [2].

In fig. 1, we show snapshots of the spatial profiles of several thermodynamic variables around the moment of minimum bubble radius for $P_{\mathrm{a}}=1.325 \mathrm{~atm}$, which is a typical driving pressure of SBSL. We can see that a steep compressional wave is formed, which produces high temperature, pressure, and density upon reflection from the bubble center. Similar profiles are shown in fig. 2 using the van der Waals EOS without considering ionization. A comparison of figs. 1 and 2 clearly shows that the ionization leads to lower temperature in the core. We see that without ionization, the velocity is approximately linear in $r$ and the pressure is nearly uniform, while a boundary layer shows up near the bubble wall for both the density and temperature. This shows that ionization tends to steepen the compressional waves because of the reduction of temperature and hence the speed of sound in the gas. The ionized zone is quite wide, and $\mathrm{Ar}^{+}$is the only dominant ion with few $\mathrm{Ar}^{2+}$ present (solid lines in fig. 3), contrary to the strong multiple ionizations reported in an earlier work, refs. [10,11]. For all driving pressures up to the experimental upper threshold, shock waves are absent. The maximum local ionization fraction $\alpha$ is $\approx 27(7) \%$ for $P_{\mathrm{a}}=1.35(1.23) \mathrm{atm}$.

For comparison, we also compute $\alpha$ by using the Saha equation instead of the reaction equations. We found that this equilibrium approach generally gives slightly less ionization (dotted line in fig. 3). For example, $\alpha \approx 21 \%$ for $P_{\mathrm{a}}=1.325 \mathrm{~atm}$. This suggests that the 

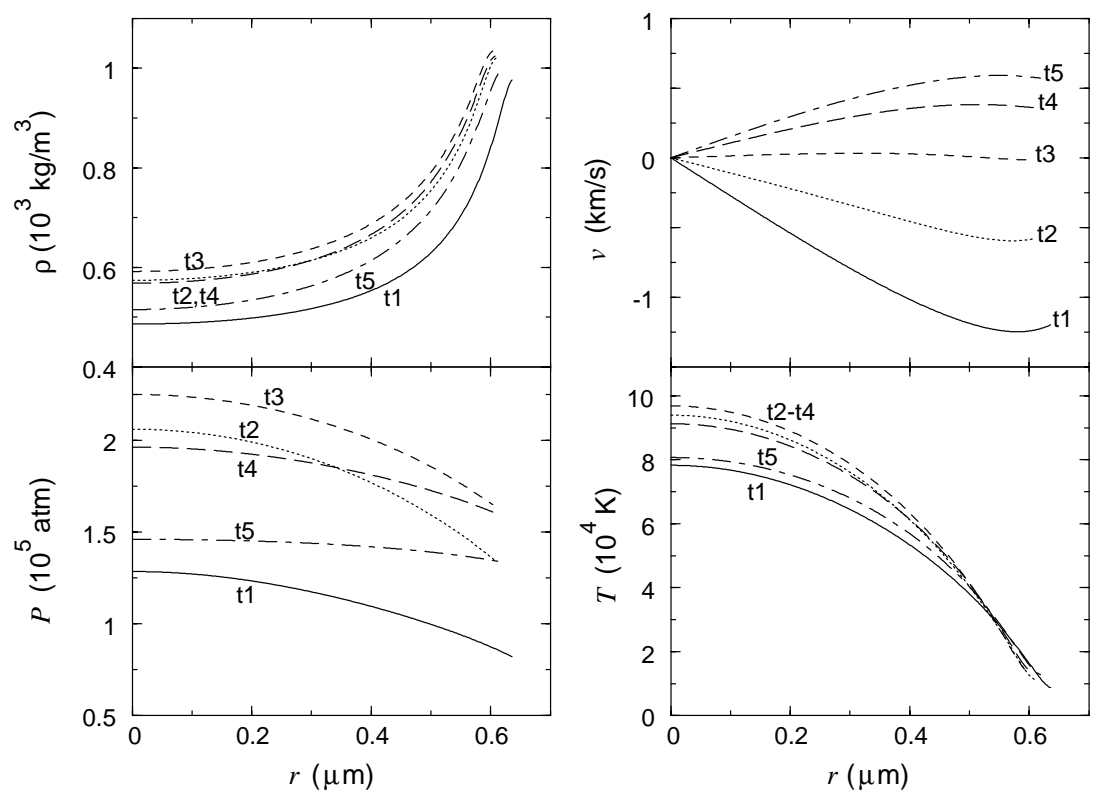

Fig. 2 - Same as fig. 1 , but without considering ionization. Here, $t 1=-45 \mathrm{ps}, t 2=-21 \mathrm{ps}, t 3=1 \mathrm{ps}$, $t 4=21 \mathrm{ps}, t 5=42 \mathrm{ps} ; t=0$ refers to the time that the bubble attains its minimum radius.

time scale of collisional ionization is shorter than that of recombinations in SBSL conditions.

To study the effects of surface tension, heat conductivity and viscosity, a simulation was done with these effects neglected. The results are dramatically different from those we have presented. High temperatures are reached, shock waves are formed, and the atoms are strongly ionized. Near the bubble center, multiple ionization up to $\mathrm{Ar}^{5+}$ (dot-dashed line in fig. 3) is found; these are consistent with refs. $[10,11]$.

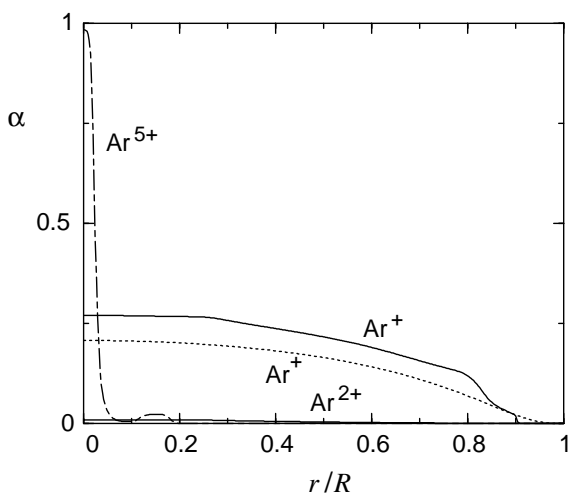

Fig. 3 - Snapshots of the spatial profiles of the ratios of $\mathrm{Ar}^{+}$and $\mathrm{Ar}^{2+}$ densities to gas density around the time that the bubble attains its minimum radius (solid lines). Ionization calculated with the Saha equation is also shown as the dotted line. For comparison, a large fraction of $\mathrm{Ar}^{5+}$ is produced when transport effects are ignored (dash-dotted line). $P_{\mathrm{a}}=1.325 \mathrm{~atm}$ and $R_{0}=4.7 \mu \mathrm{m}$ were assumed. 


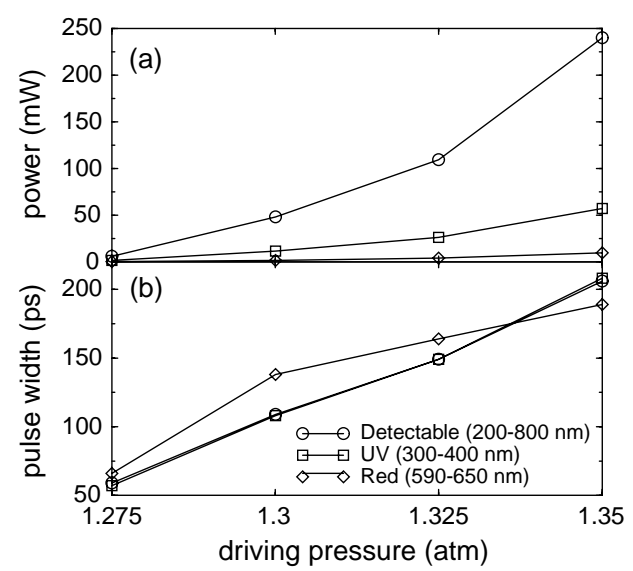

Fig. 4

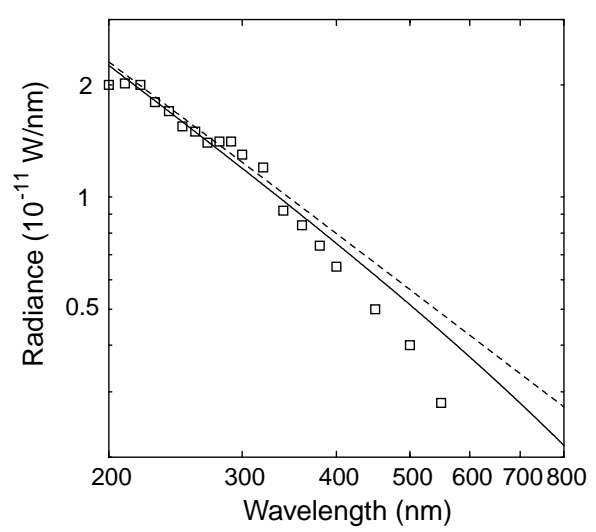

Fig. 5

Fig. 4 - Opacity-corrected blackbody radiation (a) power and (b) pulse widths vs. driving pressure. The contributions of the "red" (590-650nm) and "UV" (300-400 nm) light are shown separately.

Fig. 5 - Blackbody spectrum with (solid line) and without (dashed line) absorption correction, compared to experimental data (squares) [2]. $R_{0}$ and $P_{\mathrm{a}}$ are fitted to be $2.6 \mu \mathrm{m}$ and 1.263 atm, which is in the stable rectified diffusive regime. The spectral shapes are similar for other $P_{\mathrm{a}}$ and $R_{0}$ in the stable SL regime.

We show in fig. 4 the emitted power and pulse widths as a function of the driving pressure, which agree with experimental data in both their magnitudes and trends [3-5]. We note that the fall time of the emitted power is longer than the rise time, which is consistent with data [28], and the duration is generally longer that those from shock wave models $[6-8,10,12]$, but in better agreement with recent data [3-5].

A light emission model should be able to reproduce the observed wavelength dependence of the emitted power and pulse widths. The basic blackbody model is inadequate for SBSL because it gives rise to a larger pulse width for red light than blue light. This is because the higher temperature needed to emit blue light is reached for a shorter duration. This problem is solved in our calculation by taking into account the wavelength-dependent absorption in the outer shells, thereby suppressing the width of the red-light pulse preferentially. We show in fig. 4(b) the widths of the "red" (590-650 nm) and "UV" light (300-400 nm) separately as a function of the driving pressure. The similarity in the pulse widths for different colors [3-5], especially at high driving pressure, is nicely reproduced in our simple model, confirming the importance of the wavelength-dependent absorption [13]. The resulting spectrum agrees well with the experimental data, as shown in fig. 5, where we also show the spectrum without considering absorption effects (dashed line). It is clear that red light is affected most severely.

Several effects not considered here may be important for a realistic simulation of SBSL. We have ignored the presence of water vapors, which may play a role in SBSL [29,30]. We also have not taken into account possible modifications of the various reaction rates due to the high pressure at SBSL condition. Another high-pressure effect that we have left out is the possibility of solidification of air, which may lead to a substantial change of the EOS.

In conclusion, we have shown that transport effects suppress the formation of shock waves, and the hydro-thermal processes in SBSL are dominated by the formation of compression waves and up to $27 \%$ of first-level ionization, which in turn strengthens the compression waves. 
The existence of a partially ionized core greatly increases the opacity in an SL bubble, and the opacity-corrected blackbody power and pulse widths, and their wavelength dependence, are in excellent agreement with experimental data.

We acknowledge the support of a Hong Kong Research Grants Council grant CUHK 312/96P and a Chinese University Direct Grant (Project ID: 2060093). LY is also supported by National Key program for developing basic sciences G1999032801.

\section{REFERENCES}

[1] Gaitan D. F. et al., J. Acoust. Soc. Am., 91 (1992) 3166.

[2] Barber B. P. et al., Phys. Rep., 281 (1997) 65.

[3] Gompf B. et al., Phys. Rev. Lett., 79 (1997) 1405.

[4] Hiller R. A., Putterman S. J. and Weninger K. R., Phys. Rev. Lett., 80 (1998) 1090.

[5] Moran M. J. and Sweider D., Phys. Rev. Lett., 80 (1998) 4987.

[6] Wu C. C. and Roberts P. H., Phys. Rev. Lett., 70 (1993) 3424.

[7] Moss W. et al., Phys. Fluids, 6 (1994) 2979.

[8] Moss W. et al., Science, 276 (1997) 1398.

[9] Jarman P., J. Acoust. Soc. Am., 32 (1960) 1459.

[10] Xu N., Wang L. and Hu X. W., Phys. Rev. E, 57 (1998) 1615.

[11] Xu N., Wang L. and Hu X. W., Phys. Rev. Lett., 83 (1999) 2441.

[12] Moss W. et al., Phys. Rev. E, 59 (1999) 2986.

[13] Hilgenfeldt S., Grossmann S. and Lohse D., Nature, 398 (1999) 402.

[14] Chu M.-C. and Leung D., J. Phys. Condens. Matter, 9 (1997) 3387.

[15] Vuong V. Q. and Szeri A. J., Phys. Fluids, 8 (1996) 2354.

[16] Vuong V. Q., Szeri A. J. and Young D. A., Phys. Fluids, 11 (1999) 10.

[17] Yuan L., Cheng H. Y., Chu M.-C. and Leung P. T., Phys. Rev. E, 57 (1998) 4265.

[18] Cheng H. Y., Chu M.-C., Leung P. T. and Yunn L., Phys. Rev. E, 58 (1998) R2705.

[19] Lohse D. et al., Phys. Rev. Lett., 78 (1997) 1359.

[20] Hahn Y., Rep. Prog. Phys., 60 (1997) 691.

[21] Erdas A. and Quradi P., Z. Phys. D, 28 (1993) 185.

[22] Kamath V. and Prosperetti A., J. Acoust. Soc. Am., 85 (1987) 1538.

[23] Prosperetti A. and Lezzi A., J. Fluid Mech., 168 (1986) 457.

[24] Ho C. Y. et al., Chinese University Preprint, 2001.

[25] Chen T. W., Leung P. T. and Chu M.-C., Phys. Rev. E, 62 (2000) 6584.

[26] Zeldovich Y. B. and Raizer Y. P., Physics of Shock Waves and High-Temperature Hydrodynamic Phenomena, Vols. I and II (Academic Press, New York and London) 1966.

[27] Hilgenfeldt S., Lohse D. and Brenner M. P., Phys. Fluids, 8 (1996) 2808.

[28] Pecha R., Gomph B., Nick G. and Eisenmenger W., Phys. Rev. Lett., 81 (1998) 717.

[29] Storey B. D. and Szeri A. J., Proc. R. Soc. London, Ser. A, 456 (2000) 1685.

[30] Tögel R., Gompf B., Pecha R. and Lohse D., Phys. Rev. Lett., 85 (2000) 3165. 